\title{
GeV Gamma-Ray Astronomy in the Era of GLAST
}

\author{
Neil Gehrels \\ On behalf of the GLAST Science Team
}

NASA/GSFC Greenbelt, $M D$

\begin{abstract}
The Gamma Ray Large Area Space Telescope (GLAST) is a high energy astronomy mission planned for launch in 2005. GLAST features two instruments; the Large Area Telescope (LAT) operating from $20 \mathrm{MeV}-300 \mathrm{GeV}$ and the Gamma-ray Burst Monitor (GBM) operating from $10 \mathrm{keV}-25 \mathrm{MeV}$. GLAST observations will contribute to our understanding of active galactic nuclei and their jets, gamma-ray bursts, extragalactic and galactic diffuse emissions, dark matter, supernova remnants, pulsars, and the unidentified high energy gamma-ray sources. The LAT sensitivity is $4 \times 10^{-9}$ photons $\mathrm{cm}^{-2} \mathrm{~s}^{-1}(>100 \mathrm{MeV})$ for a one year all-sky survey, which is a factor of $>20$ better than CGRO/EGRET. GLAST spectral observations of gamma-ray bursts cover over 6 orders of magnitude in energy thanks to the context observations of the GBM. The upper end of the LAT energy range merges with the low energy end of ground-based observatories to provide a remarkable new perspective on particle acceleration in the Universe.
\end{abstract}

\section{INTRODUCTION}

The SAS-2 [1], COS-B [2] and especially CGRO/EGRET [3] experiments are the predecessors of GLAST, exploring the Universe to $\mathrm{GeV}$ energies. EGRET performed the first sensitive survey of the full gamma-ray sky between April 1991 and November 1992. Subsequent observations were based upon peer-reviewed proposals, with the instrument operating at full duty cycle until 1996 then at reduced duty cycle and reduced field of view to conserve spark chamber gas until the CGRO de-orbit in June 2000. A map of the point sources in the 3rd EGRET catalog [4] is shown in Figure 1. Major EGRET discoveries include:

- Detecting 170 unidentified sources compared to 20 previously known

- Establishing blazars as a main class of gamma-ray sources

- Detecting prompt and prolonged GeV emissions from GRBs

- Detection of LMC diffuse emission supporting a galactic origin of cosmic rays 
TABLE 1. GLAST Schedule

\begin{tabular}{ll}
\hline Item & Date \\
\hline Instrument technology NASA Research Announcement & January 1998 \\
Technology teams selected & March 1998 \\
GLAST NASA Announcement of Opportunity release & June 1999 \\
Instrument selection & January 2000 \\
Spacecraft procurement & 2002 \\
Launch & 2005 \\
\hline \hline
\end{tabular}

- Increasing the high energy gamma-ray pulsar population from two to six

- Teaming with ROSAT to establish Geminga as a radio quiet pulsar

- Studying the cosmic diffuse background with unprecedented accuracy

- Finding prolonged $\mathrm{GeV}$ emission from solar flares

Even at energies above $1 \mathrm{GeV}$, there are about 90 sources detected by EGRET [5]. Unfortunately the dropoff in EGRET sensitivity at high energies leaves the

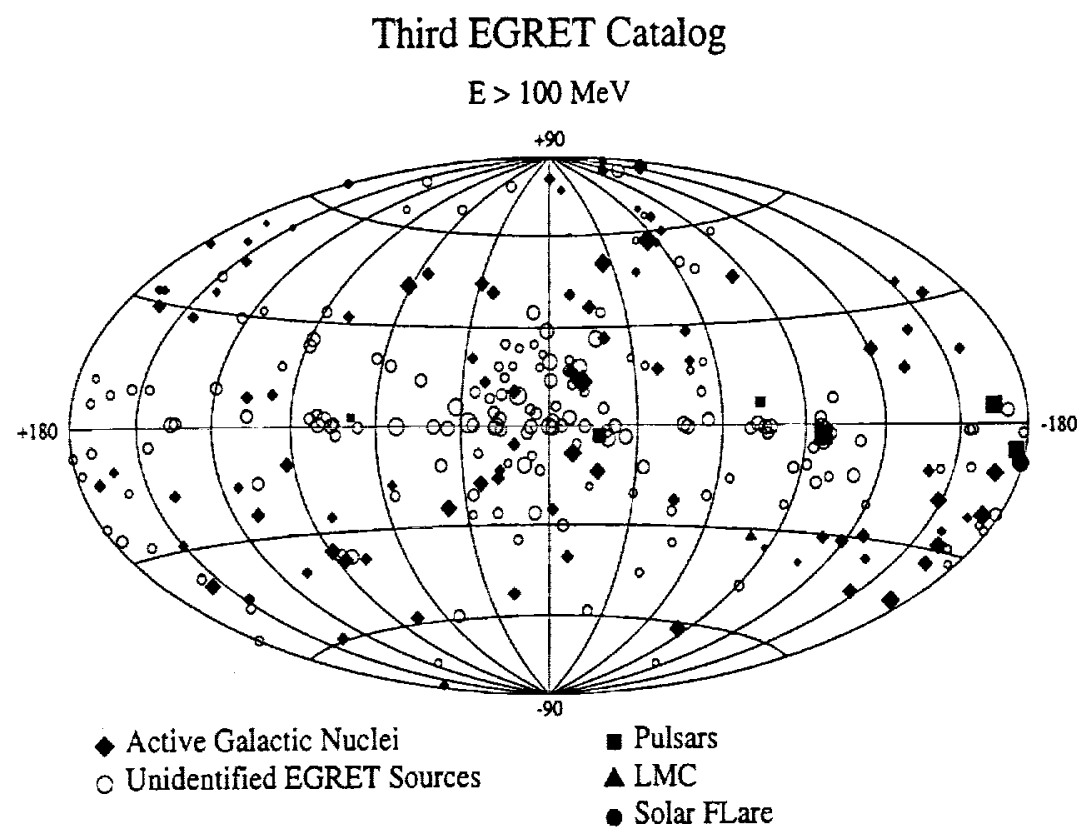

FIGURE 1. All-sky plot of the 271 sources in the 3rd EGRET Catalog 
TABLE 2. LAT instrument parameters compared to EGRET

\begin{tabular}{lll}
\hline & & \\
\hline Parameter & EGRET & GLAST/LAT \\
\hline Energy Range (MeV) & $20-30000$ & $20-300000$ \\
Energy Resolution & 0.1 & 0.1 \\
Effective Area $\left(\mathrm{cm}^{2}\right)$ & 1500 & 12000 \\
Field of View $(\mathrm{sr})$ & 0.5 & 2.5 \\
Angular Resolution $(100 \mathrm{MeV})$ & $5.8^{\circ}$ & $\sim 3.5^{\circ}$ \\
Angular Resolution $(10 \mathrm{GeV})$ & $0.5^{\circ}$ & $\sim 0.1^{\circ}$ \\
Sensitivity $\left(\mathrm{cm}^{-2} \mathrm{~s}^{-1}>100 \mathrm{MeV}\right)$ & $\sim 10^{-7}$ & $\sim 4 \times 10^{-9}$ \\
Source Location Accuracy & 5 to 30 arcmin & $<1$ arcmin \\
Mass (kg) & 1810 & 3000 \\
Lifetime & $1991-1997$ & $2005-2010$ \\
\hline
\end{tabular}

${ }^{a}$ sensitivity for one year survey at high latitudes

composition of the gamma-ray sky above $10 \mathrm{GeV}$ to our imaginations. GLAST will bridge the gap with $\mathrm{TeV}$ telescopes by performing a survey of unprecedented sensitivity to several hundred $\mathrm{GeV}$ - well beyond the limits of EGRET.

\section{GLAST MISSION}

Current NASA and Department of Energy plans call for a GLAST launch in 2005. Several of the most important GLAST mission milestones are listed in Table 1. Scientific development of the mission is led by a Science Working Group. Instrument selection for both the pair-production telescope (LAT) and secondary instrument (GBM) are now complete.

A science support center for guest investigator support will be located at the Goddard Space Flight Center. In addition, as part of the GLAST project four Independent Data Scientists (IDS) were chosen. These GLAST associates contribute technical and scientific advice to help ensure that the mission achieves its scientific goals. The scientists and their fields of expertise are: C. Dermer (NRL) - nonthermal universe, B. Dingus (Wisconsin) - transients, M. Pohl (Ruhr U.) - diffuse galactic, S. Thorsett (UCSC) - pulsars.

The primary instrument onboard GLAST is the LAT, a solid-state pairconversion telescope being developed by a team led by Stanford University (PI P. Michelson). The secondary instrument is the GBM being developed by a team led by Marshall Space Flight Center (PI: C. Meegan).

\section{Large Area Telescope}

The LAT, as shown in Figure 2, has the typical elements of a pair-conversion telescope. The main component of the LAT is a tracker/converter which converts 


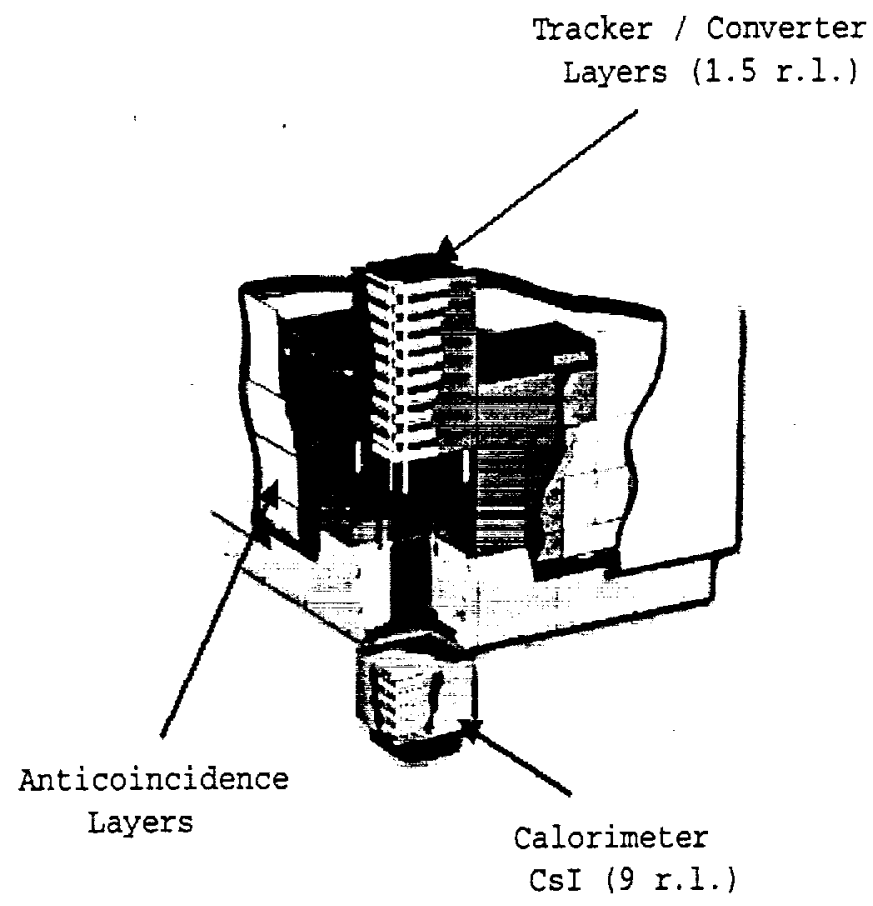

FIGURE 2. Schematic view of the Large Area Telescope showing the principal telescope components.

the incident cosmic gamma-ray to an electron/positron pair. The paths of these and subsequent particles are then followed by particle tracking detectors. The GLAST LAT uses pairs of thin silicon strip detectors for the tracker in combination with lead converter sheets. The LAT has a modular design, with 19 individual trackers set in a $4 \times 4$ array. The total thickness of each tracker section is about 1.4 radiation lengths. Unlike past pair-conversion telescopes which used spark chamber technology, the LAT has no consumables. This design provides excellent efficiency, reliability, and resolution.

Directly below the tracker is a 9 radiation length calorimeter consisting of segmented arrays of $\mathrm{CsI}(\mathrm{Tl})$ crystals. The conversion of particle energies to scintillations in the crystals is the central component of energy measurement for each cosmic gamma-ray. The segmentation of the calorimeter provides additional information on the distribution and development of the shower initiatated in the tracker.

An anticoincidence shield surrounds each tracker. This shield is made of segmented plastic scintillator tiles. Vetoing cosmic ray events by the anticoincidence shield is crucial for reducing the otherwise overwhelming charged particle background.

The LAT capabilities are compared to those of EGRET and the upcoming AGILE project [6] in Table 1. The GLAST mission will be flown in low-Earth orbit and 


\begin{tabular}{|c|c|c|}
\hline Parameter & BATSE & GBM \\
\hline Energy Range (MeV) & $\begin{array}{l}0.025-1.9 \text { (LAD) } \\
0.007-10 \text { (SD) }\end{array}$ & $\begin{array}{l}0.01-1 \text { (LED) } \\
0.15-25 \text { (HED) }\end{array}$ \\
\hline Detectors & $\mathrm{NaI}$ & $\mathrm{NaI} / \mathrm{BGO}$ \\
\hline FOV & Full Sky & $8.6 \mathrm{sr}$ \\
\hline Location Accuracy & $2-5^{\circ}$ & $1.5-3^{\circ}$ \\
\hline Burst Sensitivity $\left(\mathrm{cm}^{-2} \mathrm{~s}^{-1}\right)$ & 0.2 & 0.6 \\
\hline
\end{tabular}

will operate in both a zenith pointing mode and a stare mode. The instrument has a huge field-of-view; $>16 \%$ of the sky at a time, and in zenith pointing mode scans $\sim 75 \%$ of the sky every orbit. Combining this field of view with the large effective area and good angular resolution, gives a sensitivity of $4 \times 10^{-9}$ photons $\mathrm{cm}^{-2} \mathrm{~s}^{-1}$ (>100 MeV) for a one year all-sky survey. This is at least a factor of 20 improvement over EGRET. Other outstanding features of the LAT are the large energy range (4 decades in energy including the currently unexplored region greater than $10 \mathrm{GeV}$ ), and the unprecedented angular resolution for gamma rays (factor of 3 or more better than EGRET).

\section{Gamma-ray Burst Monitor}

The GBM is the secondary instrument onbaard GLAST. It was chosen to complement the LAT in the particularly difficult area of gamma-ray burst studies. Specifically, the GBM enables GRB spectroscopy down to $\mathrm{keV}$ energies and provides GRB trigger and position information to the LAT. The GBM capabilities are compared to CGRO/BATSE in Table 3.

Twelve NaI detectors operating from $10 \mathrm{keV}$ to $1 \mathrm{MeV}$ and two BGO detectors operating from $150 \mathrm{keV}$ to $25 \mathrm{MeV}$ comprise the GBM. The separate NaI detectors are oriented to provide a large $(8 \mathrm{sr})$ field-of-view. This field, being larger than the LAT field, allows the GBM to direct repointing of the spacecraft to either center or target GRBs. The BGO detectors are not involved in the GRB trigger, but supply extended spectral coverage which extends to the lower end of the LAT range.

The spectral information provided by the GBM is especially critical. GRB's emit most of their energy around or below $1 \mathrm{MeV}$. The high-resolution spectral observations made by the GBM, when combined with LAT information, provides spectral observations over 6 orders of magnitude in energy. This GRB spectral coverage is unmatched by any other space mission. 


\section{GLAST SCIENCE}

GLAST builds on the legacy of exploration established by EGRET. More than $70 \mathrm{AGN}$ have now been detected, almost all of which fall in the blazar class [4]. The prevailing view that blazars have jets aimed toward us indicates that the gamma radiation originates from particles accelerated in the jets. For pulsars, EGRET tripled the number (from 2 to 6) of spin-down pulsars detected in high energy gamma-rays. In conjunction with ROSAT [7], EGRET [8] found that Geminga is a gamma-ray pulsar with little or no radio emission. Concerning transients, EGRET made the important discovery of long lasting high energy emission from both gamma-ray bursts [9] and solar flares [10]. EGRET has also seen an eight-fold increase in the number of unidentified sources. GLAST will build on these results and open a large area of new discovery space for all gamma-ray source classes. In the following sections we discuss some of scientific topics that GLAST will address, especially at the highest energies.

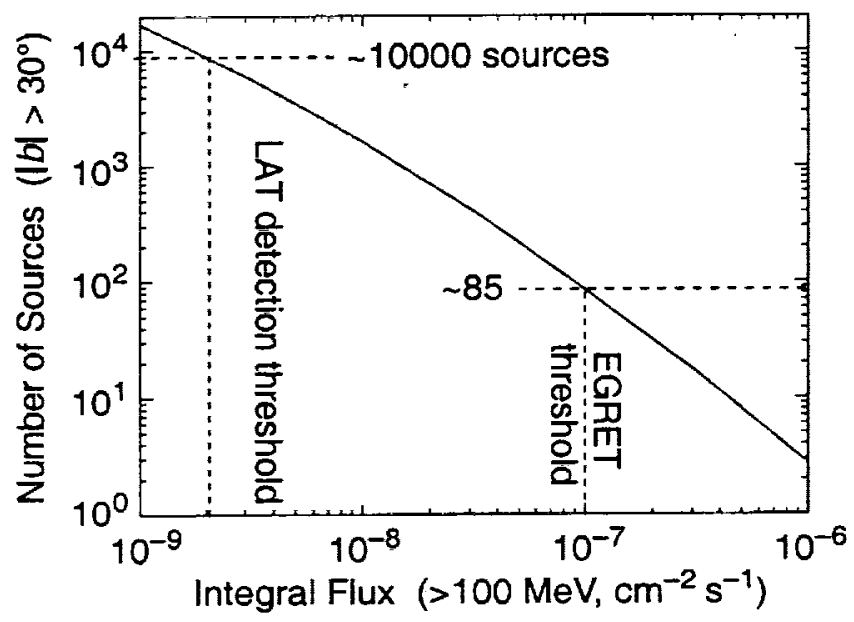

FIGURE 3. LogN-LogS plot for estimating the GLAST detection rate of AGN. The curved line is calculated assuming that the luminosity function of gamma-ray blazars is proportional to that of radio blazars and is based upon the EGRET sample. The instrument thresholds show that the number of gamma-ray blazars may grow by two orders of magnitude with GLAST. 


\section{Active Galactic Nuclei}

Most of the high latitude sources that EGRET sees are blazars, that is AGN identified at other wavelengths to be either flat spectrum radio quasars (FSRQs) or BL Lacs. They cover the distance range from $z=0.031$ to 2.3 , generally similar in distance distribution to the radio blazars. EGRET has shown that blazars can produce copious quantities of gamma rays, most likely from Compton upscattering in their relativistic particle jets [11]. Beaming factors of $\sim 10$ are required to allow the gamma rays to escape without $\gamma \gamma \rightarrow \mathrm{e}^{+} \mathrm{e}^{-}$absorption $[12,13]$. The peak in the $\nu F_{\nu}$ spectral energy distribution is typically seen to be in the high energy gammaray band.

Particularly important for studying the time varying spectrum of blazars has been multiwavelength coverage from radio through $\mathrm{TeV}$ gamma rays (e.g., [14]). From these we have an indication that the BL Lac subclass of blazars has a different characteristic multiwavelength spectrum than that of FSRQs. With the current poor statistics and small sample from EGRET and the ground-based $\mathrm{TeV}$ observatories, it is difficult to model the spectra in detail and to understand differences in subclasses. GLAST, combined with new-generation $\mathrm{TeV}$ instruments such as VERITAS, will tremendously improve blazar spectral studies, filling in the band from $20 \mathrm{MeV}$ to $10 \mathrm{TeV}$ with high significance data for hundreds of AGN.

EGRET has found that the gamma-ray emission from blazars is highly variable and usually detects blazars when they flare. In almost all blazars seen, the emission is detected in only one or two observations and not seen in other good exposures. GLAST will have the capability to monitor most AGN in the sky at all times with its wide field of view. Also, its high sensitivity will allow the first observations of low-state emission from blazars. Based on the $\log \mathrm{N}-\operatorname{logS}$ plot shown in Figure 3, the number of AGN that GLAST will detect at $5 \sigma$ is $\sim 10000$.

\section{Extragalactic Background Light}

High energy photons propagating through intergalactic space are attenuated by photon-photon pair production interactions with the intergalactic infrared-opticalUV radiation field (extragalactic background light or EBL). The origin of the lower-energy intergalactic photons is starlight, predominantly from starburst activity during the epoch of galaxy formation. The effect of this attenuation may be discernible in the ground-based TeV observation of Mrk 421 [15]. The GLAST sensitivity and spectral resolution at energies beyond $10 \mathrm{GeV}$ will establish a large database of accurate spectra as a function of blazar distance. The combination of GLAST and future ground-based instruments will be a powerful tool for systematically studying the attenuation of AGN spectra and thereby measuring the EBL to redshifts of $z \sim 4$. 


\section{Gamma Ray Bursts}

A key result from EGRET is that GRBs can have high energy (> $50 \mathrm{MeV}$ ) emission during the burst, extending to $\mathrm{GeV}$ energies and lasting for about an hour afterward $[9,16]$. The remarkable observation of GRB 940217, for instance, detected high energy radiation for about 6000 seconds (interrupted by an Earth occultation). A very high energy photon at $18 \mathrm{GeV}$ is seen at 4700 seconds. The presence of high energy gamma rays during and after bursts is of key importance in understanding the acceleration mechanisms during the burst and as the blastwave interacts with its surroundings. Although the EGRET findings are intriguing, they provide poor statistics with only 4 bursts detected during the main event and 1 burst detected in long-lasting afterglow. EGRET also suffered from saturation effects, a problem the very low LAT deadtime $(\leqslant 1 \mathrm{~ms})$ alleviates.

GLAST will significantly expand this data set by detecting $\sim 100$ bursts/yr with the LAT and GBM combined. Understanding GRB spectra and their relation to other parameters like duration, host, and afterglow properties requires accurate determinations of the low and high energy power law indices and the relevant break energies. By providing time resolved spectra from the classic GRB detection range around a hundred $\mathrm{keV}$ to 10 's of GeV or more, the GLAST GRB observations will be a uniquely important database.

\section{Dark Matter}

One of the leading candidates for the dark matter thought to dominate the Universe are stable, weakly-interacting massive particles (WIMPs). One candidate in supersymmetric extensions of the standard model in particle physics is the neutralino, which might annihilate into gamma rays in the $30-300 \mathrm{GeV}$ range covered by GLAST (e.g., [17]). The LAT sensitivity and spectral resolution are capable of detecting or tightly constraining the presence of such lines.

\section{Pulsars}

A number of young and middle-aged pulsars have their energy output dominated by gamma-ray emission. Because the gamma rays are directly related to the particles accelerated in the pulsar magnetospheres, they give specific information about physics in high magnetic and electric fields. Models based on the EGRET pulsars such as polar-cap [18] and outer gap models [19] make specific predictions that will be testable with the larger number of GLAST pulsars [20]. One key prediction for each model is the presence of high-energy spectral cutoffs, typically at energies beyond $1 \mathrm{GeV}$. As with AGN spectroscopy, the LAT high-energy response and spectral resolution is critical for testing these models. As illustrated in Figure 4, GLAST will detect approximately 50 radio pulsars or more and will greatly expand the search for more radio-quiet, Geminga-type pulsars. These studies will 


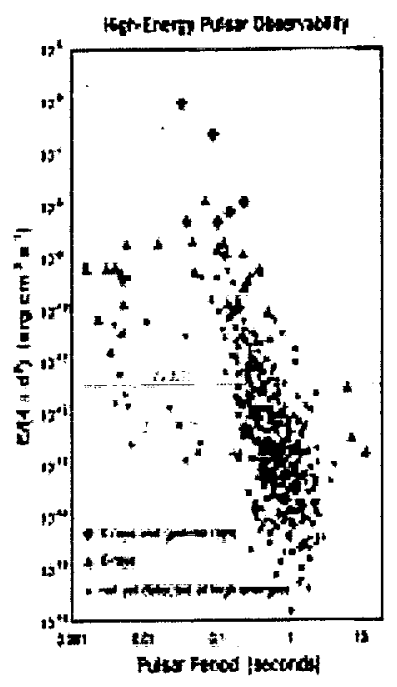

FIGURE 4. Isolated pulsar visibility in the GLAST era. The currently known high energy gamma-ray pulsars lie near the top in terms of the pulsar rotational energy loss. GLAST could increase the gamma-ray pulsar population by an order of magnitude.

lead to understanding of acceleration mechanisms in the pulsar magnetosphere and multiwavelength beaming.

\section{Supernova Remnants \& Origin of Cosmic Rays}

Although a near-consensus can be found among scientists that cosmic rays originate in supernova remnants (SNRs), the proof of that hypothesis remains elusive. $\mathrm{X}$-ray observations by ASCA give evidence for electron acceleration in SNRs such as SN1006 [21]. GLAST could observe high energy gamma rays from interactions producing pions and thereby provide the crucial observations of cosmic-ray nucleon acceleration. Some EGRET sources appear to be associated with SNRs, but the moderate spatial resolution and sensitivity make the identifications uncertain [22]. GLAST will be able to search deeply for SNRs among the unidentified EGRET sources and resolve some of those detected. Such a scenario is shown in Figure 5 for the case of $\gamma$ Cygni. The detection of an extended gamma-ray source in the vicinity of a known SNR would provide definitive evidence of cosmic ray nuclei as well as electrons.

\section{Diffuse Gamma Radiation}

Within the Galaxy, GLAST will explore diffuse radiation on scales from molecular clouds to galactic arms, measuring the product of the cosmic ray and gas densi- 


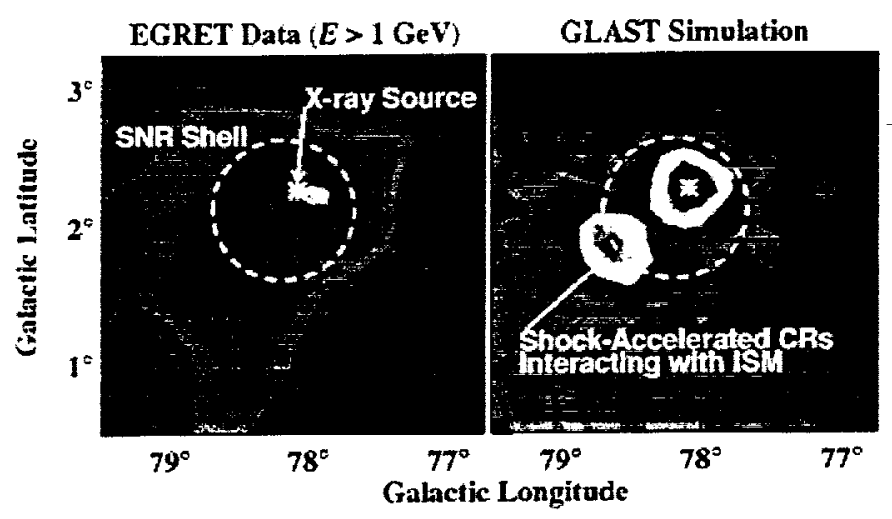

FIGURE 5. GLAST LAT simulation compared to a likelihood map of EGRET data around the $\gamma$ Cygni supernova remnant. The large circle in both frames is the EGRET location uncertainty. As shown at right, the LAT will be able to localize both point sources and regions of enhanced diffuse emission much better than before. This is especially important for studying sites and mechanisms of cosmic ray acceleration in supernova remnants.

ties [23]. The improved angular resolution and sensitivity of GLAST compared to EGRET will allow much finer detail to be mapped in the diffuse emission along the galactic plane. Also, contributions from currently undetected and unresolved point sources can be subtracted. At high latitude, GLAST will make high-sensitivity observations of the extragalactic diffuse background. With better understanding of the galactic emission, this component can be accurately subtracted. The combination of improved diffuse component observations and improved measurements of AGN will allow GLAST to determine if the extragalactic background is made up solely of unresolved AGN [24] or if there are cosmological contributions.

\section{Unidentified Sources}

There are 170 unidentified gamma-ray sources in the 3rd EGRET catalog, many of which are also detected in the $1-10 \mathrm{GeV}$ range. This source class has been a mystery since the 1970s and remain uncorrelated with known astrophysical objects. Candidates for these sources include molecular clouds [25], supernova remnants [26,22], massive stars [27], and radio-quiet pulsars [28,18,29]. Despite the rich EGRET database, only gross characteristics of this population have been determined, such as the separation of mid-latitude from low-latitude sources $[31,32]$.

EGRET source positions, typically $\approx 0.5^{\circ}$ in size, are too large for counterpart searches. The EGRET photon flux is generally too small for pulsar searches, and 

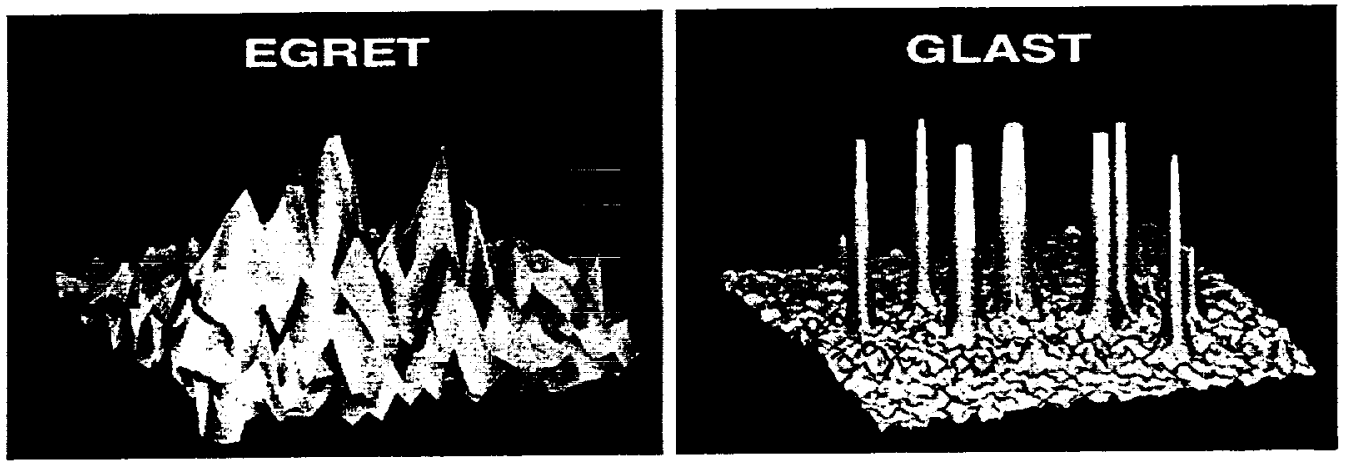

FIGURE 6. Simulation of a $15^{\circ} \times 15^{\circ}$ area in the Cygnus region. The greatly improved LAT source localization capability is critical to identifying counterparts of currently unidentified sources.

pulsar searches on strong sources have been null [30]. Both of these limitations are alleviated with GLAST. GLAST will provide much more accurate source positions, typically less than an arc-minute. Formerly source confused regions, such as that shown if Figure 6 for Cygnus, can be resolved to a much greater degree with GLAST. Individual source localizations will be much tighter than for EGRET, providing the opportunity for correlations with known objects. When combined with radio surveys and past and future X-ray observations, counterpart determination should be much more realistic.

The author gratefully acknowledge assistance from and useful discussions with D. Bertsch, E. Bloom, S. Digel, D. Macomb, P. Michelson, J. Ormes, S. Ritz and D. Thompson.

\section{REFERENCES}

1. Derdeyn, M., et al., 1972, NIM, 1998, 557.

2. Bignami, G., et al. 1975, Space Science Instrument, 1, 245.

3. Thompson, D. J., et al. 1993, ApJS, 86, 629.

4. Hartman, R. C., et al. 1999, ApJS, 123, 79

5. Macomb, D.J. \& Lamb, R.C. 2000 , ApJ, submitted

6. Barbiellini, S., et al., 2000, in GeV-TeV Gamma Ray Astrophysics Workshop: towards a major atmospheric Cherenkov detector VI, Ed. B.Dingus, M. Salamon \& D. Kieda, (New York:AIP) 467 
7. Halpern, J. P. \& Holt. S. S. 1992, Nature, 357, 222.

8. Bertsch, D., et al. 1992, Nature, 357, $30 \overline{6}$.

9. Hurley, K., et al. 1994, Nature, 372, 652.

10. Kamae, T., Ohsugi, T., Thompson, D. J., and Watanabe, K. 2000, Proceedings of the E1.1 Symposium of COSPAR Scientific Commission E, Ed. K. Makishima, L. Piro \& T. Takahashi (Pergamon Press) 905

11. Blandford, R. \& Rees, M. J. 1978, in Pittsburgh Conference on BL Lac Objects, ed. A.M. Wolfe (Pitsburg, Pittsburgh University Press) 328.

12. Mattox, J., et al. 1993, ApJ, 410, 609.

13. Dermer, C. D. \& Gehrels, N. 1995, ApJ, 447, 103.

14. Wehrle, A., et al. 1998, ApJ,197, 178.

15. Stecker, F. W. \& De Jager, O. C. 1997, ApJ, 476, 712.

16. Dingus, B. L., Catelli, J. R., and Schneid, E. J. 1997, in 25th ICRC Durban, South Africa, Vol. 3, eds. M. S. Potgieter, B. C. Raubenheiner, and D. J. van der Walt (ICRC press) 29.

17. Jungman, G., Kamionkowski, M. \& Griest, K. 1996, Phys. Reports, 267, 195.

18. Romani, R. W. 1996, ApJ, 470, 469.

19. Daugherty, J. K., \& Harding, A. K. 1996, ApJ, 458, 278.

20. Thompson, D. J., Harding, A. K., Hermsen, W., \& Ulmer, M. P. 1997, Fourth Compton Symposium, eds. C. D. Dermer, M. S. Strickman, \& J. D. Kurfess (New York: AIP) 39.

21. Koyama, K., et al., 1995, Nature, 378, 255.

22. Esposito, J. A., et al., 1996, ApJ, 461, 820.

23. Hunter, S. D., et al., 1997, ApJ, 481, 481.

24. Stecker, F. W. \& Salamon, M. H. 1996, ApJ, 464, 600.

25. Houston, B. P. \& Wolfendale, A. W. 1983, A\&A, 126, 22.

26. Sturner, S. J. \& Dermer, C. D. 1995, ApJ, 293, L17.

27. Montmerle, T. 1979, ApJ, 231, 95.

28. Thompson, D. J., et al. 1994, ApJ, 436, 229.

29. Kaaret, P. \& Cottam, J. 1996, ApJ, 462, L35.

30. Chandler, A., et al. 2000, ApJ, submitted

31. Gehrels, N., Macomb, D.J., Bertsch, D.L., Thompson, D.J. \& Hartman, R.C. 2000, Nature, 404,363

32. Grenier, I.A. \& Perrot, C. 2000, in Proceedings of the 26th International Cosmic Ray Conference, Salt Lake City, Utah, OG 2.1.11 